\title{
Segmentation and 3D Reconstruction of Microtubules in Total Internal Reflection Fluorescence Microscopy (TIRFM)
}

\author{
Stathis Hadjidemetriou ${ }^{1}$, Derek Toomre ${ }^{2}$, and James S. Duncan ${ }^{1}$ \\ 1 Departments of Diagnostic Radiology and Biomedical Engineering, \\ \{stathis, duncan\}@noodle.med.yale.edu \\ 2 Department of Cell Biology, Yale School of Medicine, New Haven, CT 06520 \\ derek.toomre@yale.edu
}

\begin{abstract}
The interaction of the microtubules with the cell cortex plays numerous critical roles in a cell. For instance, it directs vesicle delivery, and modulates membrane adhesions pivotal for cell movement as well as mitosis. Abnormal function of the microtubules is involved in cancer. An effective method to observe microtubule function adjacent to the cortex is TIRFM. To date most analysis of TIRFM images has been done by visual inspection and manual tracing. In this work we have developed a method to automatically process TIRFM images of microtubules so as to enable high throughput quantitative studies. The microtubules are extracted in terms of consecutive segments. The segments are described via Hamilton-Jacobi equations. Subsequently, the algorithm performs a limited reconstruction of the microtubules in 3D. Last, we evaluate our method with phantom as well as real TIRFM images of living cells.
\end{abstract}

\section{Introduction}

Microtubules are biopolymers of the cytoplasm. They are composed of tubulin and have a diameter of $\approx 25 \mathrm{~nm}$ [1. One of their ends interacts with the cell cortex and cell adhesions. Those interactions are pivotal for mitosis, cell migration, and active vesicle delivery [1]. In mammalian cells the other end is anchored at an organelle positioned next to the nucleus. The assembly formed provides structural stability.

Microtubules also play critical roles for cells in pathological states such as cancer. Thus, microtubules have been identified as an important target for anticancer drugs. Chemical entities such as the taxanes inhibit the polymerization of microtubules. Therefore, they stabilize the microtubule assembly and retard cancer growth. Several novel taxane derivatives are in active clinical development. It is essential to elucidate their specific effects on the microtubule assembly. Current in vivo studies are done by observation or manually [123]. Automation can enable higher throughput screening of new microtubule drugs. The latter with the goal of improving the therapeutic index with reduced toxicity.

The main objectives of analyzing microtubule data from TIRFM are to quantify their position and dynamics relative to the cortex. To this end the microtubules must be segmented, reconstructed in $3 \mathrm{D}$, and their motion must be 
tracked. Also, the state of the assembly must be expressed concisely. In this work we present an algorithm that addresses the problems of $2 \mathrm{D}$ microtubule segmentation, 3D reconstruction, and state measurement.

Some common image enhancement techniques for tubular biomedical structures have been linear filtering and morphological operations [4. The image segmentation of such structures has been implemented sequentially using local information [5 4. Tubular structures have also been segmented as minimal global paths over intensity for colon in virtual endoscopy [6]. Similarly, global minimal paths have been used to extract white matter fibers in diffusion tensor imaging [7]. Typically, global approaches to segmentation of a tubular structure require the specification of both end points of that structure 67. Some segmentation algorithms have been designed for specific microscopy techniques [89]. However, an approach for automatic processing of TIRFM images of microtubules has not been evident in the literature.

\section{Methods}

\subsection{TIRFM Image Formation and Requirements for Processing}

The substrate was living cells. They were transfected with tubulin fused with green fluorescent protein. TIRFM exploits the properties of an induced evanescent wave from a totally internally reflected laser light. It selectively illuminates and excites fluorophores in a very thin slice, $\approx 100 \mathrm{~nm}$, immediately adjacent to a glass-cell interface 10 211. Specifically, a beam of light travelling in a medium of high refractive index, such as glass with $n_{g} \approx 1.51$, enters a medium of lower refractive index, such as the cytosol of a cell with $n_{c} \approx 1.37$, beyond a certain critical angle $a_{c}$. The light is totally internally reflected and an evanescent field is induced in the cell. The exponential falloff of the evanescent field intensity does not allow fluorophores farther away from the interface to be excited. The in-vivo imaging of microtubules with TIRFM is drawn in figure 1.

A TIRFM image can be represented by the map $I: D \rightarrow \Re$, where $D \in \Re^{2}$ is the domain of dimensions $(x, y)$. The image intensity of a microtubule point at depth $z(x, y)$ above the interface is 102 :

$$
I_{z}(x, y)=I_{\text {max }} \exp ^{-\left(z(x, y)-z_{\text {min }}\right) / d_{p}},
$$

Fig. 1. TIRFM excites fluorescent microtubules that lie exclusively in a thin slice $\approx 100 \mathrm{~nm}$ immediately adjacent to the glass.

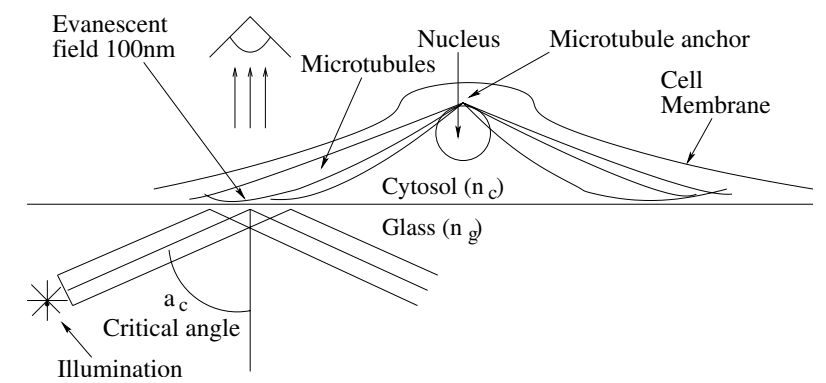


where $d_{p}$ is the penetration depth, $I_{\max }$ is the maximum image intensity, and $z_{\text {min }}$ is the corresponding depth that is an experimental constant with $z_{\min } \approx$ $10 \mathrm{~nm}$. The spatial resolution is $\approx 135 \mathrm{~nm} /$ pixel. This technique yields highcontrast images of surface events and allows the imaging of the interaction of the microtubules with the cell cortex. That interaction mediates key cellular processes such as endocytosis, exocytosis, and protein signaling [10 2 11].

The algorithm extracts a microtubule from its tip alone. The other end is not clearly distinguishable in TIRFM. The algorithm is able to extract the microtubule centerline, is robust to noise, and is robust to microtubule proximity. It can also resolve microtubule intersections. The fluorescence of isolated microtubules is used to obtain information about their $3 \mathrm{D}$ position above the interface.

\subsection{Cumulative Cost Map for Microtubule Segment}

A microtubule segment in domain $D$ of length $L$ can be represented by the curve $\mathcal{M}(s):[0, L] \rightarrow D$, where $s$ is the arc-length parameter. A microtubule segment has two boundary points $\mathcal{M}(0)=\mathbf{p}_{0}$ and $\mathcal{M}(L)=\mathbf{p}_{1}$. The set of all curves with these boundary points is $\mathcal{A}_{\mathbf{p}_{0}, \mathbf{p}_{1}}$. Every pixel at $\mathbf{p}=(x, y)$ is associated with cost $Q$ and the set of curves is associated with the cost functional $E(\mathcal{A})=\int_{0}^{L} Q\left(\mathcal{M}(s), \mathcal{M}^{\prime}(s)\right) d s$. We find the curve of minimum $E$ over the set $\mathcal{A}_{\mathbf{p}_{0}, \mathbf{p}_{1}}$. To this end we compute the minimum cost $E$ over the sets of intermediate curves $\mathcal{A}_{\mathbf{p}_{0}, \mathbf{p}}$. The result is a map of minimum cost values $U_{0}(\mathbf{p}): D \rightarrow \Re^{+}$that starts at $\mathbf{p}_{0}$. That is, $U_{0}(\mathbf{p})=\min _{\mathcal{M}(L)=p}\left\{\int_{\mathcal{M}} Q d s\right\}$. Differentiating this relation gives the Hamilton-Jacobi equation:

$$
\left\|\nabla U_{0}\right\|=Q
$$

that can be solved to obtain $U_{0}$.

The pixel cost $Q$ is the product of two factors. The first is proportional to the reverse intensity $Q_{1}=1-I(\mathbf{p}) / I_{\max }$. It is isotropic and favors microtubule fluorescence. The second factor, $Q_{2}$, is anisotropic and favors microtubule centerline. It uses directional parameters associated with every pixel, namely vectors $\mathbf{n}_{1}$ and $\mathbf{n}_{2}$, as well as scalars $r_{1}$ and $r_{2}$. The pixel cost is non-negative and given by:

$$
Q\left(\mathbf{p}, \nabla U_{0}\right)=\left(1-\frac{I(\mathbf{p})}{I_{\max }}\right) \times\left(\frac{1}{\left\|\nabla U_{0}\right\|} \sum_{i=1}^{i=2}\left|\frac{\mathbf{n}_{i} \cdot \nabla U_{0}}{r_{i}+\epsilon}\right|\right),
$$

where $\epsilon$ is a very small regularizing constant. The directional pixel parameters of $Q$ are derived from the eigenvector decomposition of the Hessian. The vectors $\mathbf{n}_{1}$ and $\mathbf{n}_{2}$ are set to the eigenvectors of the smaller eigenvalue and larger eigenvalue magnitudes, respectively. The scalars $r_{1}$ and $r_{2}$ are set to the larger and smaller eigenvalue magnitudes, respectively.

\subsection{Solution for a Cumulative Map and Curve Extraction}

The solution $U_{0}(\mathbf{p})$ of equation (2) can be expressed in terms of consecutive level sets [6]. A level set $U_{0}(\mathbf{p})=t$ is the closed curve $\mathcal{C}(v, t): \Re^{1} \rightarrow \Re^{2}$, where $v$ is 
the arc-length parameter. The family $\mathcal{C}(v, t)$ over $t$ is computed with the time evolution equation $\frac{\partial \mathcal{C}(v, t)}{\partial t}=\frac{1}{Q} \mathbf{d}(v, t)$, where $\mathbf{d}(v, t)$ is the normal to $\mathcal{C}(v, t)[$ ]. It is initialized with a curve $\mathcal{C}(., 0)$ around $\mathbf{p}_{0}$. The numerical solution visits each pixel once [12]. The retrieval of the pixels is done efficiently with a priority heap 12 and their costs $Q$ in equation (3) are minimized. This minimization is simple since $Q$ is a convex function of its parameters with a rhombus boundary [1312]. The minimum is found over directions $\mathbf{d}$ originating from the eight-connected neighborhood. Subsequently, we extract $\mathcal{M}$ by starting from $\mathbf{p}_{1}$ and proceeding along $\frac{\partial \mathcal{M}}{\partial s}=-\nabla U_{0}$. The negative discrete gradient direction of $U_{0}$ is followed to arrive at $p_{0} \in \mathcal{C}(., 0)$.

\subsection{Microtubule Segmentation in 2D}

A microtubule is extracted in terms of consecutive segments. Each segment is computed based on its neighborhood and the remaining image domain is not considered. The neighborhood is formed using a local coordinate system $\left(x^{l}, y^{l}\right)$ with origin $O^{l}$. The origin for the extraction of the first segment is the microtubule tip, $\mathbf{p}_{\text {tip }}$. The valid and non-null neighborhood is within the circle $\left\|\left(x^{l}, y^{l}\right)\right\| \leq 4 w$ and a surrounding annulus is nulled. The origin $O^{l}$ for the extraction of all subsequent segments is the most recently segmented microtubule point $\mathbf{p}_{\text {top }}$. The valid neighborhood is within the circle $\left\|\left(x^{l}, y^{l}\right)\right\| \leq 4 w$. The $x^{l}$-axis is the microtubule tangent. The null level set is in front of $\mathbf{p}_{t o p}$, where $x^{l}>2 w$ and $w$ is the microtubule width. The coordinate system is shown in figure 2] Backtracking from $\mathbf{p}_{\text {tip }}$ or $\mathbf{p}_{\text {top }}$ to the null level set gives curves $\mu_{i}$.

To determine whether a curve $\mu_{i}$ is indeed a microtubule segment we compute a contrast measure across its axis. We form three zones of width $w$, an inner zone along the curve axis, and two surrounding outer zones. We compute the average intensities $g_{i n}^{i}$ of the inner zone and $g_{\text {out }}^{i}$ of the two outer zones. The ratio $c^{i}=g_{\text {in }}^{i} / g_{\text {out }}^{i}$ gives a contrast measure. If $c^{i}>1$, the curve $\mu_{i}$ is appended to the microtubule $\mathcal{M}_{\text {tot }}$, otherwise the extraction of the microtubule ends. The algorithm is repeated independently for each microtubule tip specified. A micro-

Fig. 2.

A coordinate system centered at the most recently segmented point, $p_{\text {top }}$, of the microtubule.

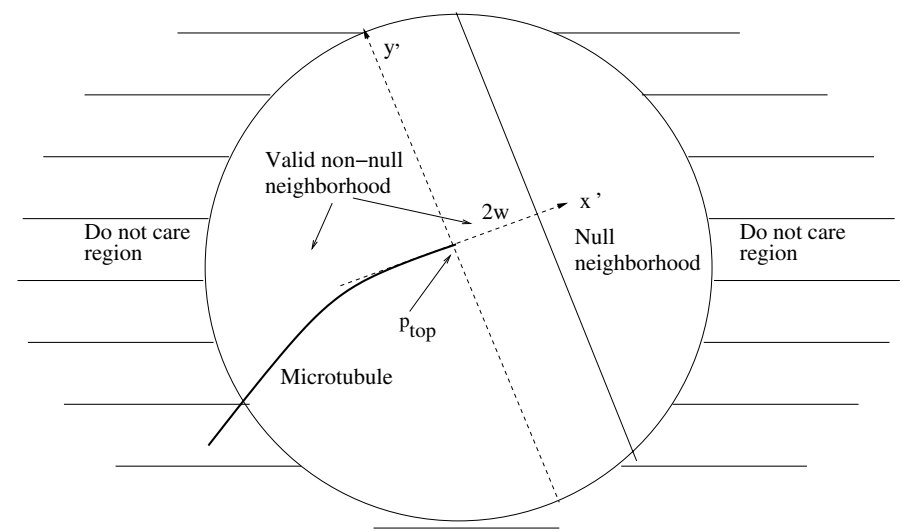


tubule consists of $\frac{L_{t o t}}{2 w}$ segments, where $L_{t o t}$ is the total microtubule length. The cost of extracting one segment from a neighborhood of size $N$ is $O(N \log N)$, where $N \leq \pi(4 w)^{2}$. Thus, the total segmentation cost is $O\left(r L_{t o t} w \log w\right)$, where $r$ is the number of microtubule tips.

\subsection{Partial Microtubule Reconstruction in 3D}

The microtubule segments close to their tips are distinct, adjacent to the glass, and have a higher signal to noise ratio. That part of the microtubules is used to perform a partial 3D reconstruction. The TIRFM formula in equation (1) is inverted to give the depth coordinate $z(x, y)=z_{\min }+d_{p} \log \left(I_{\max } / I_{z}(x, y)\right)$ relative to $z_{\min }$ [210]. Subsequently, the $3 \mathrm{D}$ data points are mean normalized and subjected to eigenvector analysis. The eigenvector of the largest eigenvalue is the microtubule tip tangent. Finally, statistics of these measures over all the microtubules are computed.

\section{Experiments on Microtubule Extraction}

We tested the sensitivity of the algorithm using parameterized sets of phantom images of microtubules with natural shapes. The phantom data was generated based on equation (11) with $d_{p}=15 \mathrm{~nm}$ and $z_{m i n}=0$. To model the degradation caused by TIRFM the microtubules were first smoothed with a Gaussian filter of standard deviation equal to two pixels and then corrupted with Gaussian noise. The ratio of the maximum intensity of the phantom microtubules to the standard deviation of the Gaussian noise gives the signal to noise ratio $(S N R)$. The noise for the phantom images is $S N R=100$ unless otherwise stated. The cross section of a microtubule is assumed to be Gaussian. The width within one standard deviation of the peak is the effective microtubule width. The phantom microtubules have $w \approx 5$. The size of the phantom images is $150 \times 150$.

To evaluate the algorithm we measured the total sum of the intensities corresponding to the segmented microtubules as well as the average error in the elevation estimates. Both were plotted as a function of the phantom set parameter in figure 4. We first tested the sensitivity of microtubule segmentation using a phantom set of images with noise in the range $S N R=5-50$. An example is in figures 3 (a-c). In figure 4 (a) the $2 \mathrm{D}$ segmentation is robust to noise. The elevation estimate in figure 4 (b), however, is more sensitive.

The sensitivity of the algorithm with respect to the proximity between neighboring microtubules was also tested. We used images containing five phantom microtubules each. The distance between consecutive microtubules is $\delta=5-14$ pixels. In figure 3 (d) that distance is 6 pixels. The biggest part of the microtubules was segmented successfully as shown in figure 3 (e). The $2 \mathrm{D}$ segmentation errors in figure 4 (c) are large only for $\delta \leq w$. The elevation errors in figure 4 (d) are not affected by proximity because the initial microtubule segments have a high SNR.

Subsequently, we used sinusoidal microtubules to test the sensitivity of the algorithm to curvature. The amplitudes of the microtubules are in the range $A=$ 


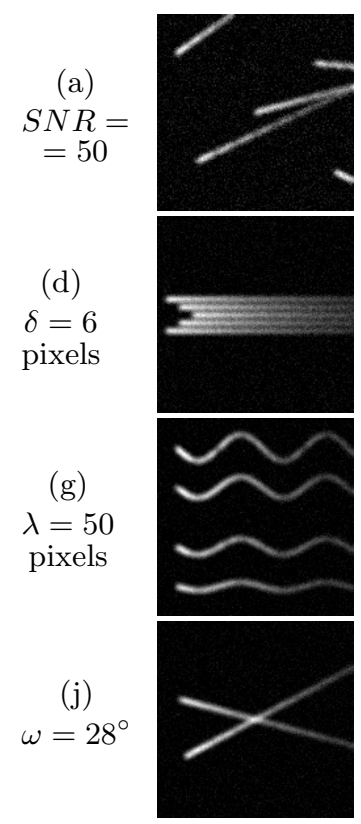

(b)

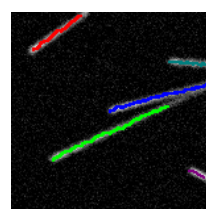

(e)

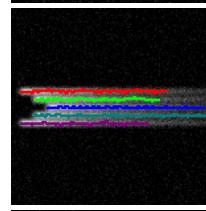

(h)

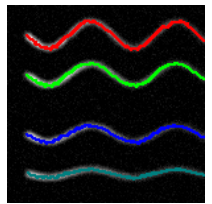

(k)

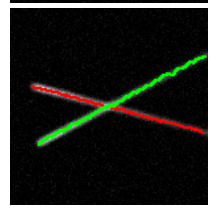

(c)

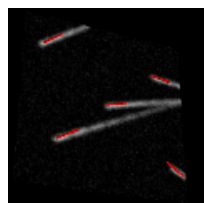

(f)

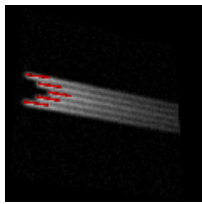

(i)

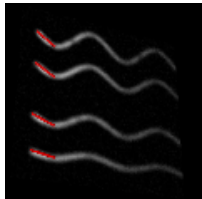

(1)

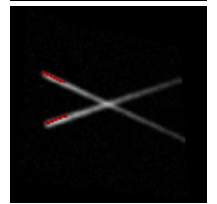

Fig. 3. In the left column are the phantom images. In the middle column are the $2 \mathrm{D}$ segmentations in color. The right column shows the 3D tip tangents in red.

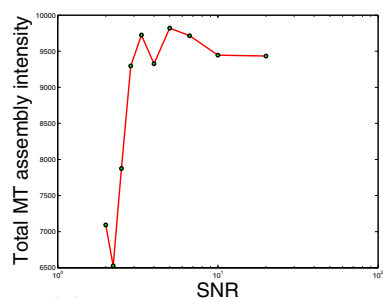

(a) Total segmentation.

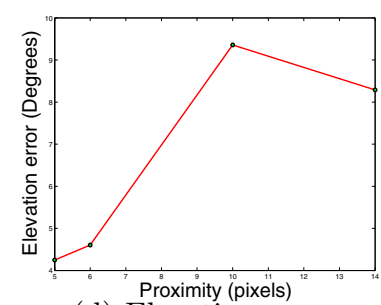

(d) Elevation error.

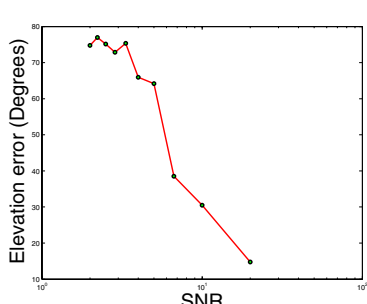

(b) Elevation error.

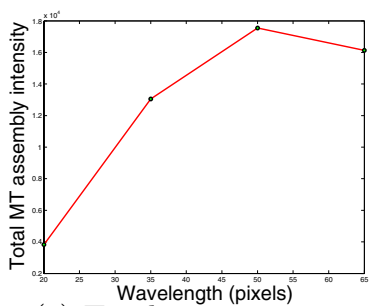

(e) Total segmentation.

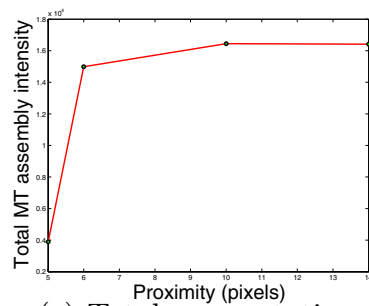

(c) Total segmentation.

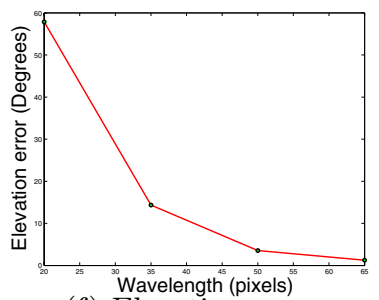

(f) Elevation error.

Fig. 4. Evaluation plots as a function of the corresponding phantom set parameter

$3-10$ pixels in each image. The wavelength takes values $\lambda=20-65$ pixels across the set. An example is in figures 3 (g-i). The plots in figures 4 (e-f) show that 
Table 1. Statistics of microtubules for the real data

\begin{tabular}{|l|l|r|c|c|c|c|c|}
\hline Image & Size & $\begin{array}{c}d_{p} \\
(\mathrm{~nm})\end{array}$ & \multicolumn{2}{|c|}{$\begin{array}{c}\text { Elevation } \\
(\text { Degrees })\end{array}$} & \multicolumn{2}{|c|}{$\begin{array}{c}\text { Depth } \\
(\mathrm{nm})\end{array}$} & $\begin{array}{c}\text { Evaluation } \\
\text { error (pixels) }\end{array}$ \\
\hline \hline & & & Mean & St. dev. & Mean & St. dev. & \\
\hline \hline figure 5 5 (a) & $179 \times 150$ & 45 & $0.24^{\circ}$ & $0.23^{\circ}$ & 42.7 & 16.0 & 1.6 \\
\hline figure 5 (d) & $205 \times 140$ & 165 & $1.11^{\circ}$ & $0.70^{\circ}$ & 70.0 & 37.0 & 1.5 \\
\hline figure 5(g) & $125 \times 56$ & 45 & $0.20^{\circ}$ & $0.05^{\circ}$ & 8.5 & 6.6 & 1.3 \\
\hline
\end{tabular}

the algorithm fails only for very high curvature. The last set of phantom images tested the sensitivity of the algorithm with respect to microtubule intersections. The resolution of microtubules succeeds for intersection angles $\omega \geq 28^{\circ}$. This is shown in figures $3(\mathrm{j})$ and $(\mathrm{k})$.

The algorithm was used to analyze several images of living epithelial cells acquired with TIRFM. Some examples are shown in the left column of figure 5. The images in figure 5 are in reverse intensity to improve visualization. The extracted microtubules are shown next to them in the same row drawn with different colors. The images in the right column show a viewpoint of the $3 \mathrm{D}$ tangents of the microtubules at their tips in red. The data and reconstruction statistics are in table 1 .

(a)

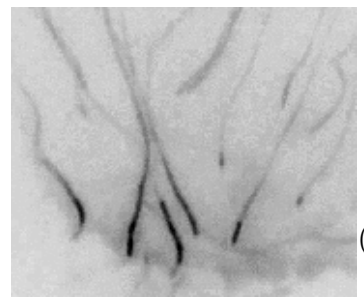

(b)

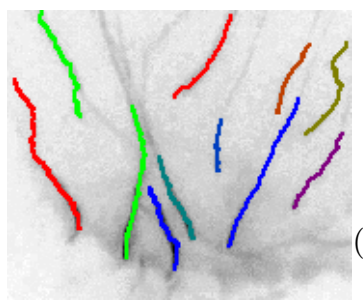

(c)

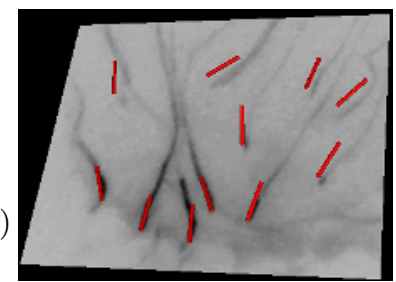

(d)

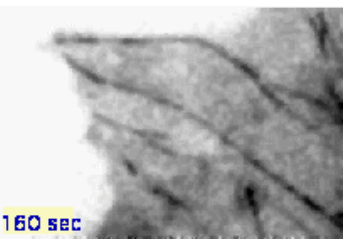

(g)

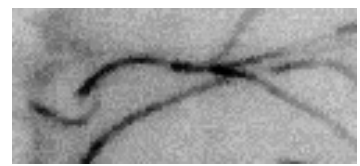

(h)

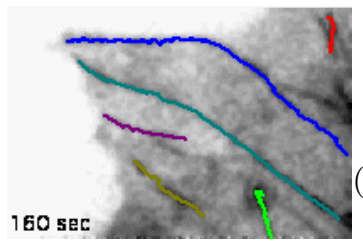

(f)
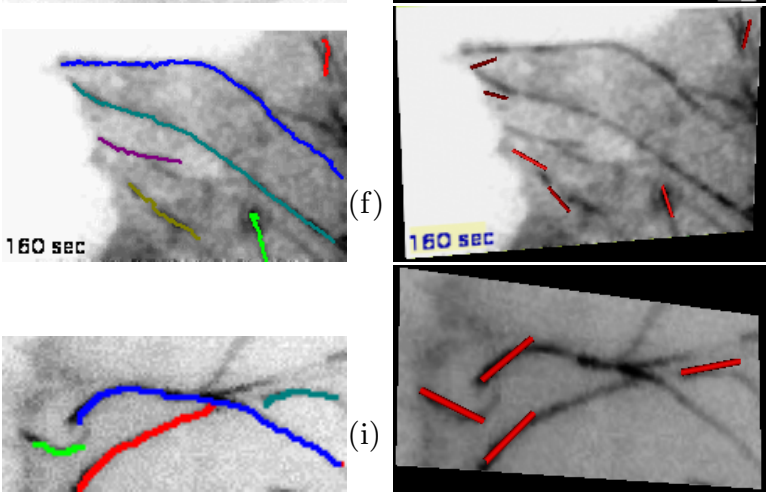

(i)

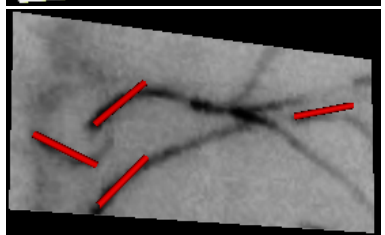

Fig. 5. The data are in the left column. The next column shows the segmented microtubules in color, and the right column shows the $3 \mathrm{D}$ tip tangents in red. 
The 2D segmentation was evaluated with ten manually traced microtubule points starting from their tips. The error for each specified point was its lowest distance from the segmented centerline of that microtubule. The total segmentation error was the average error over the specified points. The errors for the segmentations shown in figure 5 (b), figure 5 (e), and figure 5 (h) are in table 1 . The low error demonstrates the accuracy of the extraction of the microtubule centerline. The experiments were performed with a Xeon CPU. The average total computation time of the algorithm for each phantom image was $\approx 29 \mathrm{sec}$, and for the real data it was $\approx 3 \min 13 \mathrm{sec}$. The low run time demonstrates the efficiency of the algorithm.

\section{Discussion}

The interaction of the microtubules with the cell cortex is pivotal for cell physiology. The microtubules are also involved in cell pathological states such as cancer. TIRFM is a convenient method to examine the critical region close to the cell cortex. We have developed a semi-automatic algorithm for high throughput quantitative study of TIRFM images of microtubules. It first extracts microtubules in $2 \mathrm{D}$ in terms of consecutive segments. The extraction of the segments is robust to noise and along their centerlines. The sequential extraction of the microtubules allows microtubule intersections, the extraction from a single starting point, as well as robustness to microtubule proximity. Subsequently, the algorithm implements a 3D tip tangent and depth reconstruction. The algorithm has been applied to phantom as well as real data. It was shown to be efficient and accurate.

\section{References}

1. Desai, A., Mitchison, T.: Microtubule polymerization dynamics. Annu. Rev. Cell Dev. Biol. 13 (1997) 83-117

2. Krylyshkina, O., Anderson, K., Kaverina, I., Upmann, I., Manstein, D., Small, J., Toomre, D.: Nanometer targeting of microtubules to focal adhesions. The Journal of Cell Biology 61 (2003) 853-859

3. Waterman-Storer, C., Salmon, W., Salmon, E.: Feedback interactions between cell-cell adherens junctions and cytoskeletal dynamics in newt lung epithelial cells. Molecular Biology of the Cell 11 (2000) 2471-2483

4. Hadjidemetriou, S., Duncan, J., Toomre, D., Tuck, D.: Automatic quantification of microtubule dynamics. In: Proc. of IEEE ISBI. (2004) 656-659

5. Noordmans, H., Smeulders, A.: High accuracy tracking of 2D/3D curved linestructures by consecutive cross-section matching. Pattern Recognition Letters 19 (1998) 97-111

6. Deschamps, T., Cohen, L.: Fast extraction of minimal paths in 3D images and applications to virtual endoscopy. Med. Image Analysis 5 (2001) 281-299

7. Parker, C., Wheeler-Kingshott, C., Barker, G.: Estimating distributed anatomical connectivity using fast marching methods and diffusion tensor imaging. IEEE Trans. in Medical Imaging 21 (2002) 505-512 
8. Danuser, G., Tran, P., Salmon, E.: Tracking differential interference contrast diffraction line images with nanometre sensitivity. Journal of Microscopy 198 (1999) 34-53

9. Ponti, A., Vallotton, P., Salmon, W., C.M.Waterman-Storer, Danuser, G.: Computational analysis of f-actin turnover in cortical actin meshworks using fluorescent speckle microscopy. Biophysical Journal 84 (2003) 3336-3352

10. Axelrod, D.: Total internal reflection fluorescence at biological surfaces, in Noninvasive techniques in cell biology. Wiley-Liss, New York, New York, Inc. (1990)

11. Toomre, D., Manstein, D.: Lighting up the cell surface with evanescent wave microscopy. Trends in Cell Biology 11 (2001) 298-303

12. Sethian, J., Vladimirsky, A.: Ordered upwind methods for static Hamilton-Jacobi equations: Theory and algorithms. SIAM J. Numer. Anal. 41 (2003) 325-363

13. Lin, Q.: Enhancement, Extraction, and Visualization of 3D Volume Data. Thesis Licentiate, Dep. of Electrical Engineering, Linkoping University (2001) 